\title{
Thermal concurrence mixing in a one-dimensional Ising model
}

\author{
D. Gunlycke, V. M. Kendon, and V. Vedral \\ Optics Section, Blackett Laboratory, Imperial College, London SW7 2BW, United Kingdom
}

S. Bose

Centre for Quantum Computation, Clarendon Laboratory, Parks Road, Oxford OX1 3PU, United Kingdom

(Received 8 March 2001; published 6 September 2001)

\begin{abstract}
We investigate the entanglement arising naturally in a one-dimensional Ising chain in a magnetic field in an arbitrary direction. We find that for different temperatures, different orientations of the magnetic field give maximum entanglement. In the high-temperature limit, this optimal orientation corresponds to the magnetic field being perpendicular to the Ising orientation ( $z$ direction). In the low-temperature limit, we find that varying the angle of the magnetic field very slightly from the $z$ direction leads to a rapid rise in entanglement. We also find that the orientation of the magnetic field for maximum entanglement varies with the field amplitude. Furthermore, we have derived a simple rule for the mixing of concurrences (a measure of entanglement) due to the mixing of pure states satisfying certain conditions.
\end{abstract}

DOI: $10.1103 /$ PhysRevA.64.042302

PACS number(s): 03.67.-a, 75.10.Jm

\section{INTRODUCTION}

Entanglement, the rather counterintuitive nonlocal correlations exhibited by quantum systems, has recently become one of the most valuable resources in quantum information processing [1]. Over the past few years, it has developed into a quantifiable physical resource [2-4] in an analogous manner to energy. Thus, the amount of entanglement present naturally in complex physical systems (systems with many interacting components) now becomes a relevant question to ask. Condensed-matter physicists have long investigated correlations between parts of composite systems. Entanglement is the quantum or nonlocal part of these correlations. As such, it can behave very differently from the total correlations. For example, while correlations are averaged on mixing states, entanglement generally decreases. The topic of variation of entanglement in condensed-matter systems with respect to the variation of external parameters such as temperature, field components, etc., is a relatively unexplored and potentially rich area of study. In this context, as a simple initial model, Arnesen, Bose, and Vedral have studied the variation of entanglement with temperature and magnetic field in a one-dimensional (1D) isotropic finite Heisenberg chain [5]. Prior to that, Nielsen had investigated the entanglement between two qubits interacting via the Heisenberg interaction at a non-zero temperature [6] and O'Connor and Wootters have investigated the entanglement in the ground state of an antiferromagnetic isotropic Heisenberg ring [7]. In Ref. [5], the entanglement at a nonzero temperature, being that of a thermal state, was called thermal entanglement. Subsequently, Wang has studied the quantum Heisenberg $X Y$ model [8] and the two-qubit anisotropic $X X Z$ model [9] in a similar context.

In this paper, we are going to study the thermal entanglement in the 1D Ising model in an external magnetic field [10]. Ising-like interactions form the basic coupling in many proposals for experimental systems that can be used to perform quantum computation, see, for example, [11-14]. The 1D Ising model describes a set of linearly arranged spins (qubits), each interacting with its nearest neighbors by a coupling that is proportional to $\hat{\sigma}_{z} \otimes \hat{\sigma}_{z}$. This coupling can be diagonalized in a basis of disentangled states. Naively, one might think that this implies a complete absence of entanglement in the Ising model. However, an external magnetic field with a component, however small, along a direction perpendicular to the $z$ axis is sufficient to make the eigenstates entangled.

We start in Sec. II by considering, analytically, the case of two qubits interacting via the Ising interaction in a magneticfield orthogonal to the $z$ direction. In Sec. III, we formulate a theorem for the concurrence mixing due to occupation of both the ground and the excited states. Next, in Sec. IV, we consider numerically the variation of entanglement with the orientation of magnetic field. Before concluding, in Sec. V we show that the kinds of behavior found for two qubits also hold for many qubits.

\section{TWO QUBITS WITH ISING INTERACTION}

The Hamiltonian for an isotropic quantum Ising model with nearest-neighbor couplings in an external magnetic field can in the most general form be expressed as

$$
\hat{H}=J \sum_{\langle i, j\rangle} \hat{\sigma}_{z}^{i} \hat{\sigma}_{z}^{j}+\vec{B} \cdot \sum_{k} \overrightarrow{\hat{\sigma}}^{k}
$$

where the indices $i, j$, and $k$ label the $N$ spins. Here we will consider systems in one spatial dimension with periodic boundary conditions so the $N$ th spin also couples to the first spin. Thus, we have a qubit ring. First we will consider the case of $N=2$. Our Hamiltonian can then be written as

$$
\hat{H}=2 J \hat{\sigma}_{z} \otimes \hat{\sigma}_{z}+\vec{B} \cdot(\overrightarrow{\hat{\sigma}} \otimes \hat{I}+\hat{I} \otimes \overrightarrow{\hat{\sigma}}) .
$$

The usual form of the Ising model has a magnetic field only along the $z$ axis. This case has no entanglement at all, since the Hamiltonian is diagonal in the standard disentangled basis $\{|00\rangle,|01\rangle,|10\rangle,|11\rangle\}$, where $|0\rangle$ stands for spin up and 


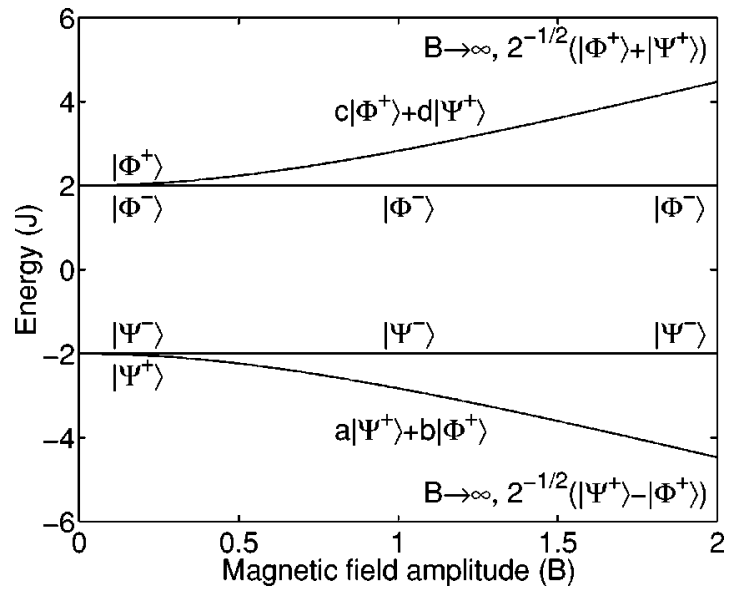

FIG. 1. Energy levels with corresponding eigenstates. The coefficients $a, b, c$, and $d$ are functions of $B$, and the states $|\Phi \pm\rangle$ $=(|00\rangle \pm|11\rangle) / \sqrt{2}$ and $|\Psi \pm\rangle=(|01\rangle \pm|10\rangle) / \sqrt{2}$ are the four Bell states.

1) stands for spin down. However, in this paper, we will consider the cases when the magnetic field is not parallel to the $z$ axis.

\section{Orthogonal fields}

Let us first study the special case when the magnetic field is perpendicular to the $z$ axis, say $\vec{B}=B \vec{x}$. Our system is now described by the following Hamiltonian:

$$
\hat{H}=2 J \hat{\sigma}_{z} \otimes \hat{\sigma}_{z}+B\left(\hat{\sigma}_{x} \otimes \hat{I}+\hat{I} \otimes \hat{\sigma}_{x}\right) .
$$

In this section, we will use $J>0$, without loss of generality [16]. We are going to investigate the entanglement in this two-qubit Ising ring. In this paper, we will use the squared concurrence $[4,15]$, called the tangle $\tau$, as a measure of entanglement. To calculate this, first we need to define the product matrix $R$ of the density-matrix $\rho$ and its spin-flipped matrix, $\tilde{\rho}=\left(\hat{\sigma}_{y} \otimes \hat{\sigma}_{y}\right) \rho^{*}\left(\hat{\sigma}_{y} \otimes \hat{\sigma}_{y}\right)$. Hence, we have

$$
R \equiv \rho \tilde{\rho}=\rho\left(\hat{\sigma}_{y} \otimes \hat{\sigma}_{y}\right) \rho^{*}\left(\hat{\sigma}_{y} \otimes \hat{\sigma}_{y}\right)
$$

Now concurrence is defined by

$$
C=\max \left\{\lambda_{1}-\lambda_{2}-\lambda_{3}-\lambda_{4}, 0\right\}
$$

where the $\lambda_{i}$ are the square roots of the eigenvalues of $R$, in decreasing order. In this method, the standard basis, $\{|00\rangle,|01\rangle,|10\rangle,|11\rangle\}$, must be used. As usual for entanglement measures, the tangle ranges from 0 (no entanglement) to 1 , when the two qubits are maximally entangled.

For finite temperatures we need the density-matrix $\rho$ for a system that is at thermal equilibrium. This is given by $\rho$ $=e^{-\hat{H} / T} / Z$, where $Z=\operatorname{tr}\left(e^{-\hat{H} / T}\right)$ is the partition function (using units where the Boltzmann constant, $\left.k_{B}=1\right)$. We then solve the time-independent Schrödinger equation for our qubits. The energy levels of our Hamiltonian (2.3) are, in rising order, $-2 \sqrt{J^{2}+B^{2}},-2 J, 2 J, 2 \sqrt{J^{2}+B^{2}}$, as in Fig. 1 .

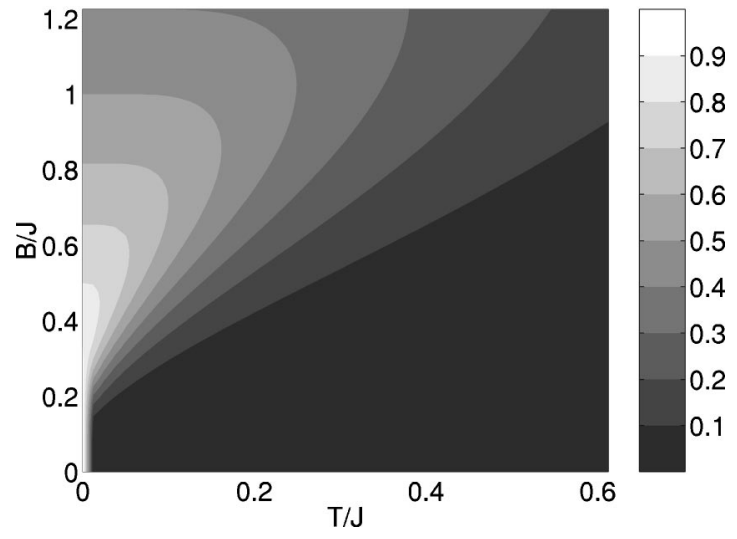

FIG. 2. Contour plot of the tangle of two qubits obeying an Ising Hamiltonian with coupling $J$, in a perpendicular magnetic field $B$, for temperatures $T$. The parameter space lies entirely within the nonclassical region, except for a thin line at $B=0$. Unfortunately, with our resolution this cannot be visualized.

For zero temperature, only the lowest-energy level is populated. The tangle of this pure state can easily be calculated from the density matrix, for $B>0$,

$$
\tau=\frac{J^{2}}{J^{2}+B^{2}}=\frac{1}{1+(B / J)^{2}} .
$$

For finite temperature, the analytical expressions are complicated, so we present them graphically in Fig. 2, a contour plot of the tangle $\tau$, as a function of magnetic-field amplitude $B$ and the temperature $T$.

From Eq. (2.6), it is clear that the entanglement is highest for nearly vanishing magnetic fields and decreases with increasing field amplitude. Physically this is because the field tends to align the qubit spins in a different disentangled state from the spin-spin coupling; it is the trade off between the field and the Ising interaction that produces the entanglement. For a strong field $(B \gg J)$ the spins will become completely aligned along the field direction, whereupon the entanglement will drop to zero. However, Eq. (2.6) is not valid for strictly $B=0$, in which limit it seems to predict maximal entanglement. At precisely $B=0$, in fact, no entanglement is present (the eigenstates are same as those of the usual Ising Hamiltonian without any magnetic field). Hence, there is a quantum phase transition [10] at the point $B=0$ when the entanglement jumps from zero to maximal even for an infinitesimal increase of $B$. As we will see later, this point is only one point on a transition line for $B$ fields along the $z$ axis.

Let us now turn our attention to the more realistic case of nonzero temperatures. For a general pure state, only one of the eigenvalues of Eq. (2.4) is nonzero and therefore equal to the tangle. This statement is proved in Lemma 1 in the next section. For low temperature and magnetic field, i.e., $B, T$ $\ll J$, it is a good approximation to assume that only the two lowest-energy levels are populated. This becomes clear when we look at Fig. 1 in the regime $B \ll J$. The lowest two levels are much closer to each other compared with their separation from the third lowest-energy level (i.e., the second excited state). Thus, when the temperature is low, only the lowest 
two levels appear in the state of the system. We will find (Theorem 1, next section) that, in our case, the combination of the two lowest states also combines their concurrences in the following weighted subtraction:

$$
C=\max \left\{\left|w_{0} C_{0}-w_{1} C_{1}\right|, 0\right\},
$$

where the index 0 refers to the ground state, while 1 refers to the excited state and $w_{0}$ and $w_{1}$ are the weights of the ground and excited states, respectively. The weights can be any weights from the statistics, for example, Maxwell-Boltzmann statistics or Fermi-Dirac statistics. We call this concurrence mixing. In our case, the first excited state is the Bell state, $\left|\Psi^{-}\right\rangle=(|01\rangle-|10\rangle) / \sqrt{2}$, which has $\tau=1$, and Eq. (2.7) reduces to

$$
C=\left|w_{0} \frac{J}{\sqrt{J^{2}+B^{2}}}-w_{1}\right| .
$$

In general, the first term in the above equation is larger than the second, and in this case the concurrence decreases with temperature as $w_{0}$ decreases and $w_{1}$ increases (c.f. Fig. 2). In Fig. 2, we also see that, for a given temperature, the entanglement can be increased by adjusting the magnetic field and is generally largest for some intermediate value of the magnetic field. This effect can be understood by noting that $w_{0}$ increases with increasing $B$ as the energy separation between the levels increase, but $J / \sqrt{J^{2}+B^{2}}$ decreases. As a result, the combined function reaches a peak as we vary $B$, and decreases subsequently, inducing analogous behavior for the concurrence.

\section{CONCURRENCE MIXING}

In this section, we are going to formulate and prove a useful concurrence mixing theorem. We begin with a lemma that illustrates the method used in the theorem. The results of Lemma 1 appear in Ref. [4].

Lemma 1. Let $\rho$ be a pure density matrix. Then the product matrix $R=\rho \tilde{\rho}$, where $\tilde{\rho}=\left(\hat{\sigma}_{y} \otimes \hat{\sigma}_{y}\right) \rho^{*}\left(\hat{\sigma}_{y} \otimes \hat{\sigma}_{y}\right)$, has only one nonzero eigenvalue, and its value is the concurrence squared, i.e., the tangle. For a general pure state, $|\alpha\rangle$ $=a|00\rangle+b|01\rangle+c|10\rangle+d|11\rangle$ the concurrence is

$$
C=2|a d-b c|
$$

or, written as a Schmidt decomposition, $C=2\left|c_{0} c_{1}\right|$, where $c_{i}$ are the two Schmidt coefficients.

Proof. Consider a general pure density-matrix $\rho=|\alpha\rangle\langle\alpha|$. By writing out the product matrix

$$
\rho \tilde{\rho}=|\alpha\rangle\left\langle\alpha\left|\hat{\sigma}_{y} \otimes \hat{\sigma}_{y}\right| \alpha\right\rangle\langle\alpha| \hat{\sigma}_{y} \otimes \hat{\sigma}_{y},
$$

we see directly that $\left|u_{0}\right\rangle=|\alpha\rangle$ is an eigenstate with eigenvalue $\left|\left\langle\alpha\left|\hat{\sigma}_{y} \otimes \hat{\sigma}_{y}\right| \alpha\right\rangle\right|^{2}$. It is always possible to find three more vectors $\left|\alpha_{k}^{\perp}\right\rangle, k=1,2,3$, all of them linearly independent of each other and orthogonal to $|\alpha\rangle$. Thus, the remaining three eigenvectors can be written as $\left|u_{k}\right\rangle=\hat{\sigma}_{y} \otimes \hat{\sigma}_{y}\left|\alpha_{k}^{\perp}\right\rangle$, $k=1,2,3$, all with eigenvalue zero. From Eq. (2.5), we now get the concurrence to be $C=\left|\left\langle\alpha\left|\hat{\sigma}_{y} \otimes \hat{\sigma}_{y}\right| \alpha\right\rangle\right|=2|a d-b c|$. Thus, for a pure state, concurrence can be defined as the absolute expectation value of the operator $\hat{\sigma}_{y} \otimes \hat{\sigma}_{y}$. By tracing out one qubit and solving for the eigenvalues, which are equal to the square of the Schmidt coefficients $c_{i}$ of the remaining density matrix, we find that the concurrence also can be written as $C=2\left|c_{0} c_{1}\right|$.

Theorem 1. Consider two pure states of the same system $\left|\alpha_{m}\right\rangle$ and $\left|\alpha_{n}\right\rangle$. If the spin-flip overlap is zero, i.e.,

$$
\left\langle\alpha_{m}\left|\hat{\sigma}_{y} \otimes \hat{\sigma}_{y}\right| \alpha_{n}\right\rangle=0,
$$

then the concurrence of the mixture of the two pure states, with weights $w_{i}$, can be expressed as

$$
C_{\text {mixed }}=\left|w_{m} C_{m}-w_{n} C_{n}\right| .
$$

Proof. Let $\rho_{i}=\left|\alpha_{i}\right\rangle\left\langle\alpha_{i}\right|, i=m, n$, be our two pure states. From our lemma, we have

$$
\begin{gathered}
\rho_{i} \tilde{\rho}_{i}\left|u_{i 0}\right\rangle=C_{i}^{2}\left|u_{i 0}\right\rangle, \\
\rho_{i} \tilde{\rho}_{i}\left|u_{i k}\right\rangle=0, \quad k=1,2,3,
\end{gathered}
$$

where $\left|u_{i 0}\right\rangle=\left|\alpha_{i}\right\rangle$ and $\left|u_{i k}\right\rangle=\hat{\sigma}_{y} \otimes \hat{\sigma}_{y}\left|\alpha_{i k}^{\perp}\right\rangle$. Let us write our mixed state $\rho$ as a weighted average of the pure density matrices $\rho=w_{m} \rho_{m}+w_{n} \rho_{n}$. Since $\tilde{\rho}$ is an antilinear transformation of $\rho$, we also have $\tilde{\rho}=w_{m} \tilde{\rho}_{m}+w_{n} \tilde{\rho}_{n}$. Using these assumptions, we can write down the product matrix

$$
\rho \tilde{\rho}=w_{m}^{2} \rho_{m} \tilde{\rho}_{m}+w_{m} w_{n}\left(\rho_{m} \tilde{\rho}_{n}+\rho_{n} \tilde{\rho}_{m}\right)+w_{n}^{2} \rho_{n} \tilde{\rho}_{n} .
$$

Our condition (3.3) makes the cross terms drop out, since

$$
\rho_{i} \tilde{\rho}_{j}=\left|\alpha_{i}\right\rangle\left\langle\alpha_{i}\left|\hat{\sigma}_{y} \otimes \hat{\sigma}_{y}\right| \alpha_{j}\right\rangle\left\langle\alpha_{j}\right| \hat{\sigma}_{y} \otimes \hat{\sigma}_{y}=0 .
$$

Furthermore, condition (3.3) together with Eq. (3.5) gives the following relation:

$\rho_{i} \tilde{\rho}_{i}\left|u_{j 0}\right\rangle=\left|\alpha_{i}\right\rangle\left\langle\alpha_{i}\left|\hat{\sigma}_{y} \otimes \hat{\sigma}_{y}\right| \alpha_{i}\right\rangle\left\langle\alpha_{i}\left|\hat{\sigma}_{y} \otimes \hat{\sigma}_{y}\right| \alpha_{j}\right\rangle=\delta_{i j} C_{j}^{2}\left|u_{j 0}\right\rangle$.

Equations (3.7)-(3.9) give two of the four eigenequations of the product matrix

$$
\rho \tilde{\rho}\left|v_{i}\right\rangle=\left(\delta_{i m} w_{m}^{2} C_{m}^{2}+\delta_{i n} w_{n}^{2} C_{n}^{2}\right)\left|v_{i}\right\rangle,
$$

where $\left|v_{i}\right\rangle=\left|u_{i 0}\right\rangle=\left|\alpha_{i}\right\rangle$. Since these two eigenvectors only span two dimensions in the four-dimensional space, we can always find another two vectors $\left|\alpha_{k}^{\perp}\right\rangle, k=2,3$ which are linear independent of each other and orthogonal to the two eigenstates. Thus, the last two eigenvectors are $\left|v_{k}\right\rangle=\hat{\sigma}_{y}$ $\otimes \hat{\sigma}_{y}\left|\alpha_{k}^{\perp}\right\rangle, k=2,3$, both with zero eigenvalue. Equation (2.5) now gives our mixed concurrence formula.

Theorem 1 applies to any system with a mixture of two pure states satisfying condition (3.3). It can easily be extended to apply to the mixing of more pure states. The requirement is then that condition (3.3) must hold for all pairs of pure states. 


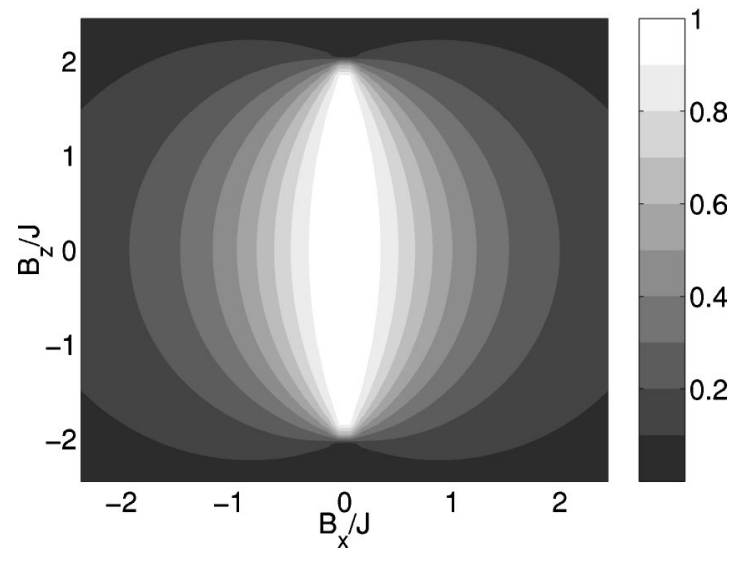

FIG. 3. Contour plot of the tangle at zero temperature in a Cartesian coordinate system. Note that the line of nonentangled states at $B_{x}=0$ for $\left|B_{z}\right|<2 J$ cannot be seen.

In our case, it would have been interesting if, when including all four levels, the concurrence could be calculated as

$$
C=\max _{k \in\{0,1,2,3\}}\left\{2 w_{k} C_{k}-\sum_{i=0}^{3} w_{i} C_{i}, 0\right\}
$$

If the highest two weights are set to zero, this expression reduces to Eq. (3.4) as expected. In fact, for a three-level approximation (involving the first three levels), Eq. (3.11) with $w_{3}=0$ is correct, however, the exact four-level concurrence is not in agreement with Eq. (3.11), because condition (3.3) does not hold for mixing the ground state with the third excited state.

\section{ARBITRARY FIELDS}

In Sec. II we treated a case of a magnetic-field orthogonal to the Ising direction. We are now going to generalize this to arbitrary magnetic fields. The new Hamiltonian can be written

$$
\begin{aligned}
\hat{H}= & 2 J \hat{\sigma}_{z} \otimes \hat{\sigma}_{z}+B(\sin \theta)\left(\hat{\sigma}_{x} \otimes \hat{I}+\hat{I} \otimes \hat{\sigma}_{x}\right) \\
& +B(\cos \theta)\left(\hat{\sigma}_{z} \otimes \hat{I}+\hat{I} \otimes \hat{\sigma}_{z}\right)
\end{aligned}
$$

where $\theta$ is the angle between the magnetic field and the Ising direction. It is sufficient to consider a variation of $B$ in a plane containing the Ising direction, because in three spatial dimensions, the Hamiltonian possesses rotational symmetry about the $z$ axis. From here and onwards we choose to specially study the antiferromagnetic case with $J>0$, since the ferromagnetic case $(J<0)$ does not have any spin-ordering competition in the $z$ direction.

The expression for the tangle is analytically solvable. However, because of a difficult cubic equation in the diagonalization, the expressions are complicated, so we present the results in graphical form. At zero temperature, Fig. 3 shows our solution when the tangle is plotted as a function of $B_{x}=B \sin \theta$ and $B_{z}=B \cos \theta$, and Fig. 4 shows the solution

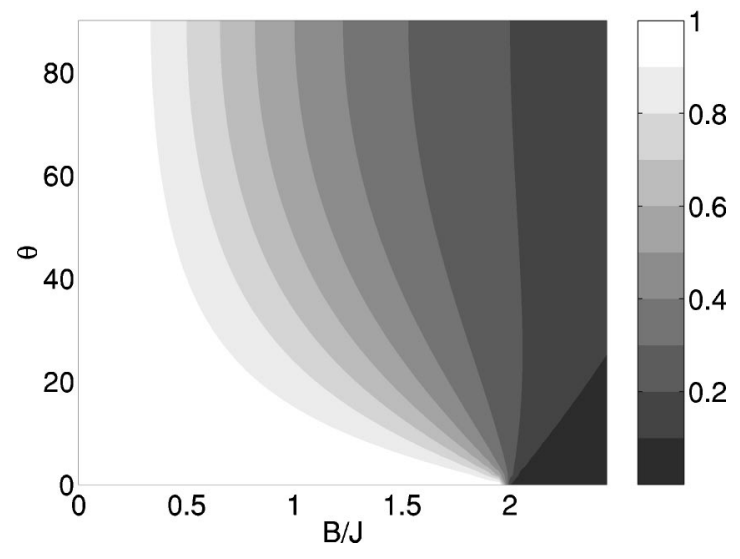

FIG. 4. Contour plot of the tangle at zero temperature in a spherical coordinate system.

when the tangle is plotted as a function of the amplitude $B$ and angle $\theta$.

We notice that the region around $B_{x}=0$ for all $\left|B_{z}\right|<2 J$ has the highest possible entanglement. At exactly at $B_{x}=0$ there should not be any entanglement, the white region of Fig. 3 indicates a quantum phase transition at $B_{x}=0$ (the sharpness of the transition being illustrated by the fact that the zero entanglement line at $B_{x}=0$ is so thin that it is invisible). For small angles $\theta$, there are two energy levels close to the energy $-2 J$ with corresponding states close to the Bell states $\left|\Psi^{ \pm}\right\rangle=(|01\rangle \pm|10\rangle) / \sqrt{(2}$. Thus, we get a maximally entangled qubit pair in the limit. However, at $\vec{B}$ $=B_{z} \vec{z}$, with $\left|B_{z}\right|<2 J$, the states are degenerate with no entanglement as a result. In the case $B_{z}>2 J\left(B_{z}<-2 J\right)$ the ground state is always the nonentangled state $|11\rangle(|00\rangle)$. Also, notice that $\theta=\pi / 2$ corresponds to our earlier orthogonal case, thus, the tangle follows Eq. (2.6). Even when $\left|B_{x}\right|$ is increased to the point where it starts to dominate, the spins will simply align along $B_{x}$ and give a disentangled state. Thus, the entanglement falls off with increasing strength of the magnetic field in either direction.

Let us now look at the case of finite temperature (thermal entanglement). The first excited state is $\left|\Psi^{-}\right\rangle$which lies at the energy $-2 J$. This state is totally independent of a magnetic field, thus, the tangle corresponding to this state forms a constant plane at one. Fortunately, condition (3.3) in our theorem is also satisfied, which makes Eq. (2.7) valid, allowing us to gain an intuitive insight into where the entanglement comes from. In Figs. 5 and 6, a numerical solution is shown at a low temperature. These plots and the following contour plots at nonzero temperatures in this paper, are calculated numerically by diagonalizing the product matrix $R$, in Eq. (2.4) and then applying Eq. (2.5). Note how fast the tangle drops to zero for a low $\left|B_{x}\right|$ component. This does not contradict the concurrence mixing formula, as the weights $w_{i}$ also depend on the magnetic field through the energy. The fast drop in the tangle is due to the degeneracy at $B_{x}=0$. The smallness of the energy difference at low values of $\left|B_{x}\right|$ makes the two levels almost equally populated even for small temperatures. The line of zero entanglement at $B_{x}=0$ for $T=0$ has broadened into a region of almost zero en- 


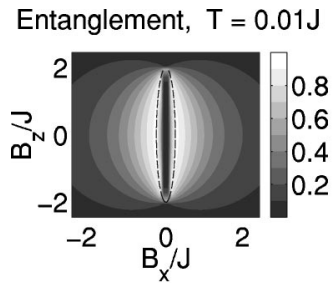

Entanglement, $\mathrm{T}=0.09 \mathrm{~J}$

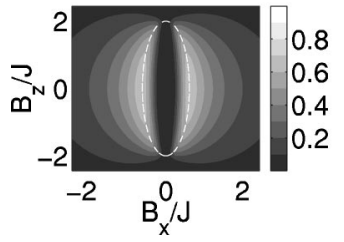

Entanglement, $\mathrm{T}=0.03 \mathrm{~J}$

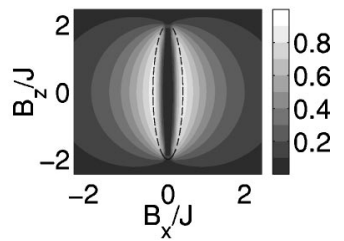

Entanglement, $T=0.27 \mathrm{~J}$

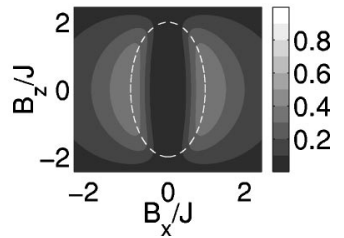

FIG. 5. Contour plots of the tangle in Cartesian coordinates for various finite temperatures, $T$. The dashed line represents an estimation of the maximum tangle using only the lowest two energy levels [cf. Eq. (4.11)].

tanglement in the finite-temperature case (compare Figs. 3 and 5).

It is also interesting to see that there exists an angle $\theta$ $=\theta^{*}(B, T)$, where the entanglement is maximum for a given temperature and amplitude. This feature can be explained heuristically if we assume that with $B_{x}$ and $B_{z}$ fixed, the entanglement should change continuously with temperature. We know that increasing the temperature widens the lowentanglement zone around $B_{x}=0$ and the entanglement has to fall off for large $\left|B_{x}\right|$. So it is expected that at some intermediate value of $\left|B_{x}\right|$ (and hence $\theta$ ) the maximal entanglement will be reached. As we increase the temperature further, the near-zero entanglement zone centered around $B_{x}=0$ widens even more and pushes the entanglement maxima away to higher and higher values of $\left|B_{x}\right|$. The highest value of the tangle tends more and more towards orthogonal fields (cf. Figs. 5 and 6). The preferred angle traverses from $\theta=0$ at zero temperature (Fig. 4) to $\theta=\pi / 2$ at $T \approx J$ (Fig. 6). In the
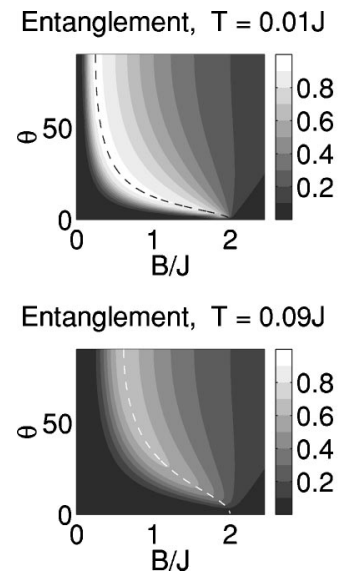

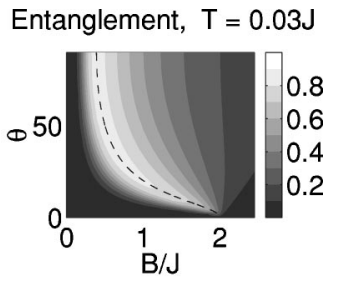

Entanglement, $\mathrm{T}=0.27 \mathrm{~J}$

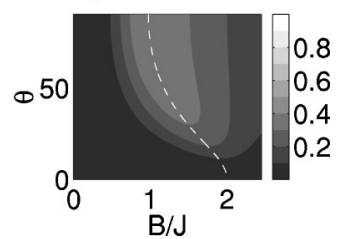

FIG. 6. Contour plots of the tangle in spherical coordinates. The angle, resulting in the maximum entanglement for a given magnetic-field amplitude, varies as a function of the amplitude and temperature. The dashed line represents an estimation of the maximum tangle using only the lowest two energy levels [cf. Eq. (4.10)].

classical limit, i.e., at very high temperatures, all entanglement fades away as expected because the state is completely mixed.

We can use the two-level approximation to get an estimate of the angle creating maximal entanglement. Let us first estimate the ground-state energy, $\epsilon$. Since we know that the lowest two energy levels are very close and the first excited state is $-2 J$, we can use the approximation $-2 J+\epsilon$ $\approx-4 J$ while solving for eigenvalues of the Hamiltonian (4.1). The ground-state energy is then

$$
\epsilon=-2 J-\frac{4 B_{x}^{2}}{4 J^{2}-B_{z}^{2}} J .
$$

For the above approximation to hold, we must operate in a region not too close to the poles at $B_{z}= \pm 2 J$. This gives the following energy difference between the two lowest levels

$$
\Delta \epsilon=-2 J-\epsilon \equiv \frac{4 B_{x}^{2}}{4 J^{2}-B_{z}^{2}} J .
$$

Unfortunately, this approximation is not good enough. In further calculations, the equations grow and soon become hopeless. So now we need to find a better energy approximation. Observe that the two terms of the ground-state energy (4.2) can be considered as the first two terms in a Taylor expansion of the kind $\sqrt{1+x}$. This suggests that we instead write

$$
\epsilon=-2 J \sqrt{1+\frac{\Delta \epsilon}{J}}=-2 J \sqrt{1+\frac{4 B_{x}^{2}}{4 J^{2}-B_{z}^{2}}} .
$$

Let us, from this equation and onwards, measure energy in units of $J$, i.e., let $J=1$. When we solve for the eigenstates and plug them into Eq. (3.1), the zero-temperatureconcurrence becomes approximately

$$
C_{0}=\frac{1}{\sqrt{1+\Delta \epsilon}}
$$

Notice that the energy approximation in Eq. (4.4) has now given us a concurrence approximation, which simplifies to the exact expression [square root of Eq. (2.6)] in the orthogonal field limit. For nonzero $B_{z}$, this formula remains an excellent approximation (as we have verified numerically).

Let us next investigate how the concurrence varies with the magnetic-field $\vec{B}$ at a fixed-finite temperature. From the concurrence mixing theorem (3.4) we get an approximation of the thermal concurrence. Recall that the first excited state is $\left|\Psi^{-}\right\rangle$with concurrence $C_{1}=1$. Thus, to find the maximum entanglement we need to solve

$$
\overrightarrow{0}=\vec{\nabla}_{\vec{B}} C=\vec{\nabla}_{\vec{B}}\left(w_{0} C_{0}-w_{1}\right)=\left(1+C_{0}\right) \vec{\nabla}_{\vec{B}} w_{0}+w_{0} \vec{\nabla}_{\vec{B}} C_{0},
$$

since $w_{1}=1-w_{0}$. If $w_{i}$ are weights following MaxwellBoltzmann statistics, we have 


$$
w_{0}=\frac{e^{-\epsilon_{0} / T}}{e^{-\epsilon_{0} / T}+e^{-\epsilon_{1} / T}} \approx \frac{1}{1+e^{-\Delta \epsilon / T}} .
$$

Inserting Eqs. (4.5) and (4.7) into Eq. (4.6) gives the following condition for maximum entanglement:

$$
T\left(1+e^{\Delta \epsilon / T}\right)=2(1+\Delta \epsilon)(1+\sqrt{1+\Delta \epsilon}) .
$$

In the region we are interested in, where temperature is not bigger than $\sim 20 \%$ of the coupling constant $J$, the temperature is also much smaller than $\Delta \epsilon$, and Eq. (4.8) can be further simplified to

$$
\Delta \epsilon=T \ln \frac{4}{T}
$$

Remember that the ground-state energy is still a function of the magnetic field. In order to fix the temperature, $\Delta \epsilon$ has to be fixed, i.e., maximum entanglement is reached in the cross section between the energy surface and the constant energy plane that follows from Eq. (4.9). From Eq. (4.3), it is clear that this is described by an ellipse in a $B_{x} B_{z}$ plane. Using the field amplitude $B$ as a parameter, the angle resulting in maximum tangle is given by

$$
\sin \theta^{*}= \pm \sqrt{\frac{\Delta \epsilon}{4-\Delta \epsilon} \frac{4-B^{2}}{B^{2}}}
$$

assuming that $|B|>\sqrt{\epsilon}$. Equation (4.10) is shown as dashed lines in Fig. 5.

Another way to parametrize the optimum line is to let $B_{z}$ be the parameter and solve for $B_{x}$,

$$
B_{x}= \pm \sqrt{\Delta \epsilon\left(1-\frac{B_{z}^{2}}{4}\right)}
$$

with $\left|B_{z}\right|<2$. This is shown as dashed lines in Fig. 6. Again, keep in mind that Eqs. (4.10) and (4.11) follow from the assumption that $\Delta \epsilon \approx 0$, and therefore the dashed lines are not valid around the poles at $B_{z}= \pm 2$ in Figs. 5 and 6 .

\section{QUBIT RINGS}

As the expressions for the thermal tangle of the general two-qubit case are already quite complicated, we cannot expect to find any easily manageable analytic expressions in the many-qubit case. Instead, we have performed numerical simulations, which gives the entanglement between neighboring qubits as shown in Fig. 7.

The behavior for even $N$ rings is quite similar to that of the two-qubit case. To understand why there is an extra lowentanglement zone around $B_{z}=0$ in the case of odd $N$ rings, one has to go back the basic cause for entanglement arising in the Ising chain. It results from the competition between the term $\hat{\sigma}_{z} \otimes \hat{\sigma}_{z}+B_{z} \hat{\sigma}_{z}$ trying to impose spin order in the $z$ direction and $B_{x} \hat{\sigma}_{x}$ trying to impose spin order in the $x$ direction. In the odd qubit case, it is impossible for all neighboring spins to be oriented oppositely, so the ordering power
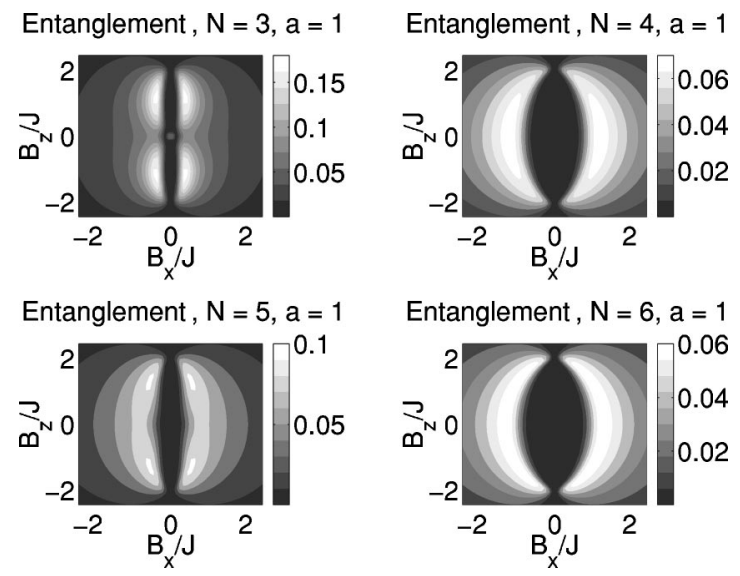

FIG. 7. Contour plots of the tangle at temperature $T=0.10 \mathrm{~J} . \mathrm{N}$ is the number of qubits in the chain and $a=1$ means that entanglement is measured between two neighboring qubits.

of $\hat{\sigma}_{z} \otimes \hat{\sigma}_{z}$ is significantly reduced. In such circumstances, it is mainly the competition between $B_{z} \hat{\sigma}_{z}$ and $B_{x} \hat{\sigma}_{x}$ (albeit aided by the small $\hat{\sigma}_{z} \otimes \hat{\sigma}_{z}$ interaction) which determines the entanglement. Thus, the high-entanglement values near $B_{z}$ $=0$ present for the two qubit (and all other even $N$ ) cases disappear for odd $N$. Note also that the entanglement in the odd $N$ case is somewhat larger in magnitude compared with the even $N$ case. This is a result of the fact that the two-terms $\hat{\sigma}_{z} \otimes \hat{\sigma}_{z}$ and $B_{z} \hat{\sigma}_{z}$ compete for the type of $z$ ordering (parallel or antiparallel neighboring spins). In the even $N$ case, this competition is much stronger and this tends to lower the entanglement by reducing the net effect of $z$-ordering terms with respect to $x$-ordering terms. As the number $N$ of qubits in the chain is increased, the difference between even and odd $N$ chains should disappear (because for large $N$, adding or removing an extra qubit from the chain should not make a significant difference). This effect is clearly seen in Fig. 7 where the difference in appearance between the plots for $N$ $=3$ and $N=4$ is much greater than that between $N=5$ and $N=6$.

Entanglement can also be calculated between nonneigh-
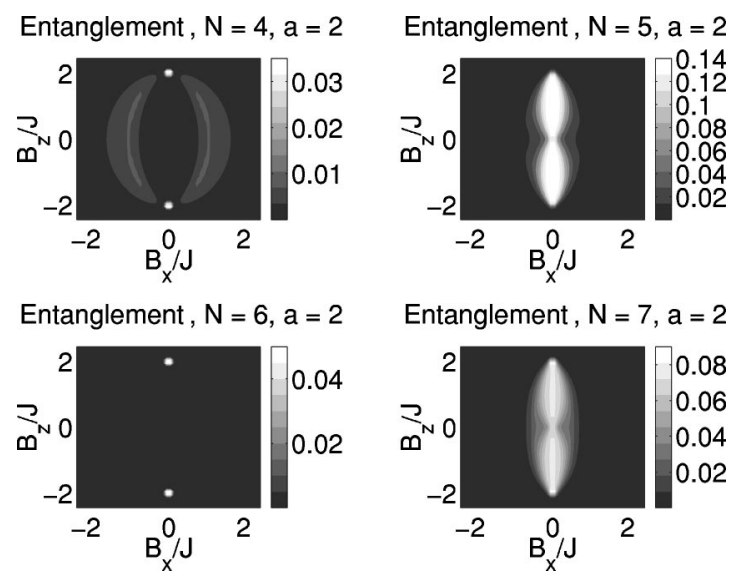

FIG. 8. Contour plots of the tangle at temperature $T=0.10 \mathrm{~J} . N$ is the number of qubits in the chain and $a=2$ means that entanglement is measured in pairs with one qubit in between. 
boring qubits with the results shown in Fig. 8 for nextnearest neighbors. Again, we observe that the even $N$ case has lower entanglement on average than the odd $N$ case. Also in the odd qubit case, the entanglement between next-nearest neighbors is somewhat complementary to that between nearest neighbors (this can be seen, for example, by placing the plots for $N=5$ in the two cases on top of each other). Thus, the amount of entanglement between pairs of nearest neighbors and pairs of next-nearest neighbors can be controlled by varying the field direction.

\section{CONCLUSIONS}

In this paper, we have investigated the natural thermal entanglement arising in an Ising ring with a magnetic field in an arbitrary direction. We have investigated two qubits analytically and three through seven qubits numerically. One of the most interesting results is the fact that for a given temperature, the (nearest neighbor, pairwise) entanglement in the ring can be maximized by rotating the magnetic field (at fixed magnitude) to an optimal direction. This can be regarded as magnetically induced entanglement. The pairwise entanglement between next-nearest neighbors can be maximized by rotating the field to a different direction. We have also proved a theorem of mixing of concurrences that is applicable to any system in which the pure states in the mixture have no spin-flip overlap.

So far, we have have only considered pairwise entanglement. In future work, we will estimate the entanglement between three or more qubits in the ring and also focus on investigating ways to detect the natural entanglement in Ising models, and on investigation of the entanglement in the large variety of available condensed-matter models of interacting systems.

\section{ACKNOWLEDGMENTS}

This work was funded by the UK Engineering and Physical Sciences Research Council and the European Union Project EQUIP (Contract No. IST-1999-11053).
[1] C.H. Bennett and D.P. DiVincenzo, Nature (London) 404, 247 (2000).

[2] V. Vedral, M.B. Plenio, M.A. Rippin, and P.L. Knight, Phys. Rev. Lett. 78, 2275 (1997); V. Vedral and M.B. Plenio, Phys. Rev. A 57, 1619 (1998).

[3] C.H. Bennett, H.J. Bernstein, S. Popescu, and B. Schumacher, Phys. Rev. A 53, 2046 (1996); S. Popescu and D. Rohrlich, ibid. 56, 3319 (1997).

[4] W.K. Wootters, Phys. Rev. Lett. 80, 2245 (1998).

[5] M.C. Arnesen, S. Bose, and V. Vedral, Phys. Rev. Lett. 87, 017901 (2001).

[6] M.A. Nielsen, Ph.D. thesis, University of New Mexico, 1998.

[7] K.M. O'Connor and W.K. Wootters, Phys. Rev. A 63, 052302 (2001); W.K. Wootters, e-print quant-ph/0001114.

[8] X. Wang, Phys. Rev. A 64, 012313 (2001).

[9] X. Wang, Phys. Lett. A 281, 101 (2001).

[10] S. Sachdev, Quantum Phase Transitions (Cambridge Univer- sity Press, Cambridge, 1999).

[11] D.G. Cory, A.F. Fahmy, and T.F. Havel, Nuclear Magnetic Resonance Spectroscopy; An Experimental Accessible Paradigm for Quantum Computing, Proceedings of the Fourth Workshop on Physics and Computation (Complex Systems Institute, Boston, MA, 1996).

[12] B.E. Kane, Nature (London) 393, 133 (1998).

[13] J.E. Mooij, T.P. Orlando, L. Levitov, L. Tian, C.H. van der Wal, and S. Lloyd, Science 285, 1036 (1999).

[14] H.J. Briegel and R. Raussendorf, Phys. Rev. Lett. 86, 910 (2001); R. Raussendorf and H.J. Briegel, ibid. 86, 5188 (2001).

[15] In this paper we only consider entanglement between pairs of qubits, tangle thus refers to the two-tangle throughout, V. Coffman, J. Kundu, and W.K. Wootters, Phys. Rev. A 61, 052306 (2000).

[16] For nonzero field $B$, substituting $J \rightarrow-J$ merely exchanges the roles of the different Bell states, as can be seen by inverting the energy axis in Fig. 1. 